\title{
Research of 5 extracts of wild Amur grape Vitis amurensis Rupr. and identification of its polyphenolic composition by tandem mass spectrometry (HPLC-MS / MS)
}

\author{
Mayya P. Razgonova ${ }^{1,2, *}$, Alexander M. Zakharenko ${ }^{1}{ }^{2}$, Irina $V$. Derbush ${ }^{1}$, Andrey $S$. \\ Sabitov $^{1}$, and Kirill S. Golokhvast ${ }^{1,2,3,4}$ \\ ${ }^{1}$ N.I. Vavilov All-Russian Institute of Plant Genetic Resources, B. Morskaya, 42-44, 190000 Saint- \\ Petersburg, Russian Federation \\ ${ }^{2}$ Far Eastern Federal University, Sukhanova, 8, 690950 Vladivostok, Russian Federation \\ ${ }^{3}$ Pacific Geographical Institute, Far Eastern Branch of the Russian Academy of Sciences, Radio, 7, \\ 690041 Vladivostok, Russian Federation \\ ${ }^{4}$ Siberian Federal Scientific Centre of Agrobiotechnology, Centralnaya, Presidium, 633501 \\ Krasnoobsk
}

\begin{abstract}
Vitis amurensis Ruprecht contains a large number of polyphenolic compounds which are biologically active components. For the most efficient and safe extraction supercritical carbon dioxide was used. In this work, for the first time, a comparative metabolomic study of biologically active substances of wild grapes collected from five different places of the Primorsky and Khabarovsk territories is carried out. To identify target analytes in ethanol extracts of grape berries, high performance liquid chromatography (HPLC) was used in combination with an amaZon SL ion trap (manufactured by BRUKER DALTONIKS, Germany) equipped with an ESI electrospray ionization source in negative and positive ion modes. The mass spectrometer was used in the scan range m/z $100-1.700$ for MS and MS / MS. Used fragmentation of the 4th order. Primary mass spectrometric results showed the presence of 89 biologically active compounds corresponding to the species $V$. amurensis, moreover, salvianolic acids F, D and G, oleanoic, ursolic, myristoleic acids, berbericinin, mearnsetin, esculin, nevadensin, stigmasterol, fucosterol, phlorizin, L-tryptophan identified for the first time in $V$. amurensis.
\end{abstract}

\section{Introduction}

Researchers attribute the appearance of the first representatives of the Vitaceae family, belonging to the genus Vitis, to the Upper Cretaceous period, when there were already types of plants that were very similar in leaves to vines. The absence of seeds does not allow, however, to have complete confidence in their belonging to the genus Vitis [1,2]. To these types, researchers include the Vitis dakotana Berry vine found in the Upper Cretaceous

\footnotetext{
*Correspondence: razgonova.mp@dvfu.ru
} 
sediments in Harding County in South Dakota, which is very similar in appearance to modern vines $[3,4]$.

The evolution of grape plants approaching the cultivated vine, judging by the fossil finds, took place especially intensively in Central and Southern Europe during the second half of the Tertiary period and then especially in the Quaternary period. On the territory of Russia, quite a lot of finds of fossils are also known belonging to the genera Cissites, Ampelopsis, Parthenocissus and especially to the genus Vitis: V. sachalinensis Krysht. and V. crenata Heer on Sakhalin, $V$. teutonica A. Br. - near Taganrog and on the Irtysh River, as well as $V$. praevinifera Sap. - on the Krynka river. All these data show that the evolution of the vine on the territory of Russia proceeded from ancient times. And now in Russia in many areas wild grapes $V$. sylvestris Gmel grow [5, 6, 7].

Very little information is available about the culture of East Asian grape varieties. $V$. lanata Roxb is cultivated in eastern India. and $V$. tomentosa Heyne, in Japan and in Korea $V$. Thunbergii Sieb. et Zucc. called V. Seiboldii hort [8]. More complete information is available regarding $V$. amurensis Rupr., which was first introduced into the culture by I.V. Michurin. In his work "Results of half a century of work" I.V. Michurin describes four forms of $V$. amurensis Rupr., which were isolated in the Far East $[9,10]$.

For the isolation of biologically active substances, ripe fruits, fruit skins, combs, leaves, seeds, vine bark, red grape wine are used. Fruits contain $65-85 \%$ water, $10-33 \%$ sugar (glucose and fructose), phlobaphene, gallic acid, quercetin, enin, glycosides monodelphinidin and didelphinidin, acids (malic, silicic, salicylic, phosphoric, tartaric, citric, etc.) pectin and tannins, potassium, magnesium, calcium, manganese, cobalt, iron and vitamins: $\mathrm{B}_{1}, \mathrm{~B}_{2}, \mathrm{~B}_{6}, \mathrm{~B}_{12}, \mathrm{~A}, \mathrm{C}, \mathrm{P}, \mathrm{PP}$, folic acid, and enzymes.

The dominant class of biologically active compounds of fruits, and especially grape ridges, are bioflavonoids and, in particular, the so-called complexes of oligomeric proanthocyanidins or condensed tannins, which are polymeric forms of flavonoids from the group of catechins [11].

In European medicine, until recently, grapes were widely used as a means of therapy and rehabilitation for a wide range of diseases: chronic recurrent inflammatory processes, tuberculosis, kidney disease, arterial hypertension, etc.

The aim of this work was a comparative metabolomic study of biologically active substances of wild grapes harvested in five different places in the Russian Far Eastern taiga in the Primorsky and Khabarovsk territories. High performance liquid chromatography (HPLC) in combination with a BRUKER DALTONIKS ion trap (tandem mass spectrometry) was used to identify target analytes in extracts. This paper presents a detailed study of the metabolomic composition of grape juice from fruits taken from five habitats of $V$. amurensis in the Far East: Pakhtusov Islands and Rikord Island (Peter the Great Bay, Sea of Japan), the vicinity of Artem (Primorsky Territory), the vicinity of the river Arsenyevka (Primorsky Territory), environs of Vyazemsky (Khabarovsk Territory).

\section{Materials and methods}

\subsection{Materials}

The object of the study was the berries of the wild grape $V$. amurensis, collected in the floodplain of the Arsenyevka River, Primorsky Territory (N. $44^{\circ} 52^{\prime} 18$ ", E $133^{\circ} 35^{\prime} 12^{\prime \prime}$; brown-gley bleached soils) in the vicinity of Vyazemsky, Khabarovsk Territory (N $47^{\circ} 32^{\prime} 15$ ", E $134^{\circ}$ 45'20"; podzolic-brown forest heavy loamy soils), in the vicinity of Artem, Primorsky Territory (St. lat. $43^{\circ} 21^{\prime} 34$ ", E $132^{\circ} 11^{\prime} 19^{\prime}$; yellow-brown earth soils), on Rikord Island, Peter the Great Bay (N $42^{\circ} 52^{\prime} 54$ ", E $131^{\circ} 40^{\prime} 06$ "; yellow-brown earth 
soils), on Pakhtusov Islands, Peter the Great Bay (N $42^{\circ}$ 53'57”, E $131^{\circ} 38$ '45 "; yellowbrown soil). The grapes were harvested at the end of August and September 2020. All samples morphologically corresponded to the pharmacopoeial standards of the State Pharmacopoeia of the Russian Federation [12].

\subsection{Methods}

\subsubsection{Fractional maceration}

To obtain highly concentrated extracts, fractional maceration was applied. In this case, the total amount of the extractant (ethyl alcohol of reagent grade) is divided into 3 parts and is consistently infused with grapes with the first part, then with the second and third. The infusion time of each part of the extractant was 7 days.

\subsubsection{High performance liquid chromatography}

To perform the separation of multicomponent mixtures, a Shimadzu LC-20 Prominence HPLC high pressure liquid chromatograph (Shimadzu, Japan) was used, equipped with a UV detector and a Shodex ODP-40 4E reverse phase column. The gradient elution program is as follows: $0.0-4 \mathrm{~min}, 100 \% \mathrm{CH}_{3} \mathrm{CN} ; 4-60 \mathrm{~min}, 100 \%-25 \% \mathrm{CH}_{3} \mathrm{CN} ; 60-75 \mathrm{~min}, 25 \%-$ $0 \% \mathrm{CH}_{3} \mathrm{CN}$; control wash $75-120$ min $0 \% \mathrm{CH} 3 \mathrm{CN}$. All HPLC analysis was done with an SPD-20A UV-VIS detector (Kanda-Nishikicho 1-chrome, Shimadzu, Chiyoda-ku, Tokyo, Japan) at $230 \eta \mathrm{m}$ and $330 \mathrm{\eta m}$; temperature $17^{\circ} \mathrm{C}$. The injection volume was $1 \mathrm{ml}$.

\subsubsection{Tandem mass spectrometry}

Mass spectrometric data were obtained using an amaZon SL ion trap (manufactured by BRUKER DALTONIKS, Germany) equipped with an ESI electrospray ionization source in negative and positive ion modes. The optimized parameters are obtained as follows: ionization source temperature: $70^{\circ} \mathrm{C}$, gas flow: $4 \mathrm{~L} / \mathrm{min}$, nebulizer gas (nebulizer): $7.3 \mathrm{psi}$, capillary voltage: $4500 \mathrm{~V}$, end plate bend voltage: $1500 \mathrm{~V}$, fragmentary: $280 \mathrm{~V}$, collision energy: $60 \mathrm{eV}$. The mass spectrometer was used in the scan range m/z $100-1.700$ for MS and MS / MS. Used fragmentation of the $4^{\text {th }}$ order.

\section{Research results}

Clarification of the metabolomic composition is an extremely important result in the system of biochemical analysis. In this work, the HPLC-MS / MS method was used with additional ionization and analysis of fragmented ions. High-precision mass spectrometric data were recorded on an AMAZON SL BRUKER DALTONIKS ion trap equipped with an ESI source in the negative / positive ion mode. A total of 300 peaks of the isolated target analytes were found on the ion chromatogram (Fig. 1). 


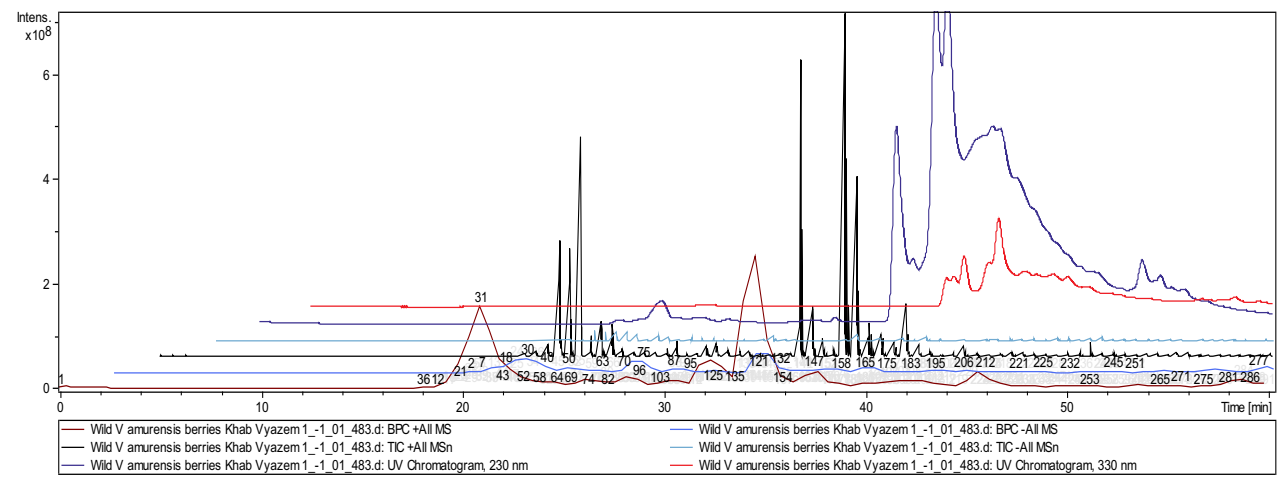

Fig. 1. Distributed graph of tandem mass spectrometry of the analyzed target analytes of the EtOH extract of $V$. amurensis (Vyazemsky, Khabarovsk Territory), represented by an ion chromatogram (Brown line - graph of the intensity of the signal of positive ions; blue line - graph of the intensity of the signal of negative ions; black line - total intensity of positive ions; blue line - total intensity of negative ions).

Based on the measurement results, a unified system table of molecular masses and fragmented ions of target analytes isolated in extracts of $V$. amurensis was compiled. (Table 1).

Table 1. Metabolome analysis of biologically active substances isolated from extracts of $V$. amurensis

\begin{tabular}{|c|c|c|c|c|c|c|c|c|}
\hline № & Identification & Formula & $\begin{array}{l}\text { Obser- } \\
\text { ved mass } \\
{[\text { [M-H]- }}\end{array}$ & $\begin{array}{c}\text { Obser- } \\
\text { ved mass } \\
{\left[\begin{array}{l}\mathrm{M}+\mathrm{H}]^{+}\end{array}\right.}\end{array}$ & $\begin{array}{c}\text { MS/MS } \\
\text { Stage 1 } \\
\text { fragmenta- } \\
\text { tion }\end{array}$ & $\begin{array}{c}\text { MS/MS } \\
\text { Stage 2 } \\
\text { fragment } \\
\text { a-tion }\end{array}$ & $\begin{array}{l}\text { MS/MS } \\
\text { Stage 2 } \\
\text { fragmen- } \\
\text { tation }\end{array}$ & References \\
\hline 1 & Malic acid & $\mathrm{C}_{4} \mathrm{H}_{6} \mathrm{O}_{5}$ & 133 & & 115 & & & $\begin{array}{c}{[22,32,57,} \\
66]\end{array}$ \\
\hline 2 & Tartaric acid & $\mathrm{C}_{4} \mathrm{H}_{6} \mathrm{O}_{6}$ & 149 & & 131 & & & {$[32,66]$} \\
\hline 3 & Umbelliferone & $\mathrm{C}_{9} \mathrm{H}_{6} \mathrm{O}_{3}$ & 161 & & 115 & & & {$[29,36]$} \\
\hline 4 & $p$-Coumaric acid & $\mathrm{C}_{9} \mathrm{H}_{8} \mathrm{O}_{3}$ & & 165 & 146 & 119 & & $\begin{array}{l}{[13,21,22,} \\
26,29,46]\end{array}$ \\
\hline 5 & Gallic acid & $\mathrm{C}_{7} \mathrm{H}_{6} \mathrm{O}_{5}$ & & 171 & 126 & & & $\begin{array}{c}{[13,20,29,} \\
36,53]\end{array}$ \\
\hline 6 & $\begin{array}{l}\text { Indole-3-carboxylic } \\
\text { acid }\end{array}$ & $\mathrm{C}_{10} \mathrm{H}_{9} \mathrm{NO}_{2}$ & & 176 & 130 & & & [50] \\
\hline 7 & Esculetin & $\mathrm{C}_{9} \mathrm{H}_{6} \mathrm{O}_{4}$ & & 179 & 133 & 115 & & {$[29,71]$} \\
\hline 8 & Caffeic acid & $\mathrm{C}_{9} \mathrm{H}_{8} \mathrm{O}_{4}$ & 179 & & 133 & & & $\begin{array}{c}{[13,15,29,} \\
46,55]\end{array}$ \\
\hline 9 & Citric acid & $\mathbf{C}_{6} \mathbf{H}_{8} \mathbf{O}_{7}$ & 191 & & $\begin{array}{l}111 ; 173 ; \\
143 ; 127\end{array}$ & & & $\begin{array}{c}{[42,57,59,} \\
66]\end{array}$ \\
\hline 10 & Quinic acid & $\mathrm{C}_{7} \mathbf{H}_{12} \mathrm{O}_{6}$ & 191 & & $111 ; 173$ & 111 & & $\begin{array}{c}{[22,29,30} \\
42,53,57, \\
61]\end{array}$ \\
\hline
\end{tabular}




\begin{tabular}{|c|c|c|c|c|c|c|c|c|}
\hline 11 & Dihydroferulic acid & $\mathrm{C}_{10} \mathrm{H}_{12} \mathrm{O}_{4}$ & 195 & & $\begin{array}{c}159 ; 129 \\
113\end{array}$ & 122 & & $\begin{array}{c}{[27,30,} \\
38]\end{array}$ \\
\hline 12 & Ethyl gallate & $\mathrm{C}_{9} \mathrm{H}_{10} \mathrm{O}_{5}$ & 197 & & 169 & 125 & & [31] \\
\hline 13 & L-Tryptophan & $\mathrm{C}_{11} \mathrm{H}_{12} \mathrm{~N}_{2} \mathrm{O}_{2}$ & & 205 & 188 & $\begin{array}{c}146 ; 170 \\
118\end{array}$ & $\begin{array}{l}144 \\
118\end{array}$ & {$[43,59]$} \\
\hline 14 & Sinapic acid & $\mathrm{C}_{11} \mathrm{H}_{12} \mathrm{O}_{5}$ & & 225 & $\begin{array}{c}179 ; 153 \\
115\end{array}$ & $133 ; 115$ & 115 & $\begin{array}{c}{[13,19,} \\
29,56, \\
57]\end{array}$ \\
\hline 15 & Myristoleic acid & $\mathrm{C}_{14} \mathrm{H}_{26} \mathrm{O}_{2}$ & & 227 & $\begin{array}{l}209 ; 181 \\
155\end{array}$ & $\begin{array}{c}199 ; 181 ; \\
127\end{array}$ & & [30] \\
\hline 16 & Resveratrol & $\mathrm{C}_{14} \mathrm{H}_{12} \mathrm{O}_{3}$ & & 229 & $142 ; 184$ & 114 & & {$[30,60]$} \\
\hline 17 & Apigenin & $\mathrm{C}_{15} \mathrm{H}_{10} \mathrm{O}_{5}$ & & 271 & $253 ; 181$ & 137 & & $\begin{array}{c}{[13,34,} \\
43,58, \\
67]\end{array}$ \\
\hline 18 & Naringenin & $\mathrm{C}_{15} \mathrm{H}_{12} \mathrm{O}_{5}$ & & 273 & $227 ; 155$ & 209 & 139 & $\begin{array}{c}{[29,53,} \\
60,62]\end{array}$ \\
\hline 19 & Linolenic acid & $\mathrm{C}_{18} \mathrm{H}_{30} \mathrm{O}_{2}$ & & 279 & $\begin{array}{c}260 ; 176 \\
120\end{array}$ & & & {$[19,71]$} \\
\hline 20 & Kaempferol & $\mathrm{C}_{15} \mathrm{H}_{10} \mathrm{O}_{6}$ & & 287 & $\begin{array}{l}269 ; 227 \\
153\end{array}$ & 153 & & $\begin{array}{l}{[29,42,} \\
45,68]\end{array}$ \\
\hline 21 & Luteolin & $\mathrm{C}_{15} \mathrm{H}_{10} \mathrm{O}_{6}$ & & 287 & $\begin{array}{l}271 ; 225 \\
\quad 175\end{array}$ & 158 & & $\begin{array}{l}{[34,43,} \\
60,67]\end{array}$ \\
\hline 22 & Dihydrokaempferol & $\mathrm{C}_{15} \mathrm{H}_{12} \mathrm{O}_{6}$ & & 289 & $\begin{array}{c}271 ; 199 \\
127\end{array}$ & $\begin{array}{c}243 ; 189 \\
118\end{array}$ & & $\begin{array}{c}{[24,41,} \\
54]\end{array}$ \\
\hline 23 & Catechin & $\mathrm{C}_{15} \mathrm{H}_{14} \mathrm{O}_{6}$ & 289 & & $245 ; 205$ & 203 & 188 & $\begin{array}{l}{[13,25,} \\
29,53, \\
57,60]\end{array}$ \\
\hline 24 & Epicatechin & $\mathrm{C}_{15} \mathrm{H}_{14} \mathrm{O}_{6}$ & & 291 & $\begin{array}{c}272 ; 175 \\
130\end{array}$ & 157 & 140 & $\begin{array}{l}{[13,29,} \\
46,53]\end{array}$ \\
\hline 25 & $\begin{array}{l}\text { 9-Oxo-10E,12Z- } \\
\text { octadecanoic acid }\end{array}$ & $\mathrm{C}_{18} \mathrm{H}_{30} \mathrm{O}_{3}$ & & 295 & $249 ; 165$ & $220 ; 125$ & & {$[30,71]$} \\
\hline 26 & Caffeoylmalic acid & $\mathrm{C}_{13} \mathrm{H}_{12} \mathrm{O}_{8}$ & 295 & & $\begin{array}{l}133 ; 179 \\
148 ; 119\end{array}$ & 115 & & [57] \\
\hline 27 & Coutaric acid & $\mathrm{C}_{13} \mathrm{H}_{12} \mathrm{O}_{8}$ & 295 & & 163 & 119 & 119 & [29] \\
\hline 28 & Kaempferide & $\mathrm{C}_{16} \mathrm{H}_{12} \mathrm{O}_{6}$ & & 301 & 283 & $\begin{array}{l}265 ; 239 ; \\
211 ; 185 ; \\
\quad 133\end{array}$ & $\begin{array}{c}211 ; \\
151\end{array}$ & $\begin{array}{l}{[45,70,} \\
74]\end{array}$ \\
\hline 29 & Ellagic acid & $\mathrm{C}_{14} \mathrm{H}_{6} \mathrm{O}_{8}$ & & 303 & $172 ; 158$ & $\begin{array}{c}144 ; 127 \\
116\end{array}$ & & $\begin{array}{l}{[29,46,} \\
52,59]\end{array}$ \\
\hline 30 & Quercetin & $\mathbf{C}_{15} \mathbf{H}_{10} \mathbf{O}_{7}$ & & 303 & $285 ; 163$ & $267 ; 159$ & 239 & $\begin{array}{l}{[13,25,} \\
29,51, \\
56,60, \\
62,68]\end{array}$ \\
\hline 31 & Hesperitin & $\mathrm{C}_{16} \mathrm{H}_{14} \mathrm{O}_{6}$ & 301 & & $257 ; 151$ & $228 ; 189$ & & $\begin{array}{c}{[16,29,} \\
60]\end{array}$ \\
\hline
\end{tabular}




\begin{tabular}{|c|c|c|c|c|c|c|c|c|}
\hline 32 & Dihydroquercetin & $\mathrm{C}_{15} \mathrm{H}_{12} \mathrm{O}_{7}$ & & 305 & $259 ; 149$ & $\begin{array}{l}199 ; \\
241 ; \\
159 \\
\end{array}$ & 171 & $\begin{array}{l}{[29,60,} \\
64]\end{array}$ \\
\hline 33 & Caftaric acid & $\mathrm{C}_{13} \mathrm{H}_{12} \mathrm{O}_{9}$ & 311 & & $149 ; 221$ & 131 & & $\begin{array}{l}{[17,22,} \\
29,55]\end{array}$ \\
\hline 34 & Salvianolic acid F & $\mathrm{C}_{17} \mathrm{H}_{14} \mathrm{O}_{6}$ & & 315 & $\begin{array}{c}269 ; 243 \\
213 ; 185 \\
144\end{array}$ & $\begin{array}{l}207 \\
181 \\
153\end{array}$ & $\begin{array}{l}179 ; \\
161 ; \\
133\end{array}$ & [22] \\
\hline 35 & $\begin{array}{l}\text { Protocatechuic acid-O- } \\
\text { hexoside }\end{array}$ & $\mathrm{C}_{13} \mathrm{H}_{16} \mathrm{O}_{9}$ & 315 & & $153 ; 298$ & 151 & & $\begin{array}{c}{[22,57,} \\
62]\end{array}$ \\
\hline 36 & $\begin{array}{l}\text { Dihydroxybenzoyl- } \\
\text { hexoside }\end{array}$ & $\mathrm{C}_{13} \mathrm{H}_{16} \mathrm{O}_{9}$ & 315 & & $153 ; 253$ & $\begin{array}{l}151 ; \\
184 \\
\end{array}$ & & [44] \\
\hline 37 & Myricetin & $\mathrm{C}_{15} \mathrm{H}_{10} \mathrm{O}_{8}$ & 317 & & $273 ; 191$ & $\begin{array}{l}255 \\
229 \\
205 \\
187 \\
163 \\
125\end{array}$ & 227 & $\begin{array}{l}{[29,30,} \\
36,51, \\
60]\end{array}$ \\
\hline 38 & Fertaric acid [Fertarate] & $\mathrm{C}_{14} \mathrm{H}_{14} \mathrm{O}_{9}$ & 325 & & $193 ; 149$ & 134 & & [29] \\
\hline 39 & $\begin{array}{l}p \text {-Coumaric acid-O- } \\
\text { hexoside }\end{array}$ & $\mathrm{C}_{15} \mathrm{H}_{18} \mathrm{O}_{8}$ & 325 & & $193 ; 163$ & 119 & & $\begin{array}{c}{[30,50,} \\
51,57, \\
62]\end{array}$ \\
\hline 40 & Galloyl glucose & $\mathrm{C}_{13} \mathrm{H}_{16} \mathrm{O}_{10}$ & 331 & & $313 ; 195$ & 166 & & [59] \\
\hline 41 & Gallic acid hexoside & $\mathrm{C}_{13} \mathrm{H}_{16} \mathrm{O}_{10}$ & 331 & & $\begin{array}{l}271 ; 169 ; \\
125\end{array}$ & 125 & & [48] \\
\hline 42 & Mearnsetin & $\mathrm{C}_{16} \mathrm{H}_{12} \mathrm{O}_{8}$ & & 333 & $\begin{array}{c}318 ; 301 ; \\
273 ; 245 \\
193 ; 165 \\
139\end{array}$ & $\begin{array}{l}301 ; \\
289 ; \\
271 ; \\
219 ; \\
192 ; \\
153 ; \\
136\end{array}$ & & [52] \\
\hline 43 & Esculin & $\mathrm{C}_{15} \mathrm{H}_{16} \mathrm{O}_{9}$ & 339 & & $177 ; 293$ & 131 & & $\begin{array}{l}{[29,30,} \\
34,71]\end{array}$ \\
\hline 44 & Salvianolic acid G & $\mathrm{C}_{18} \mathrm{H}_{12} \mathrm{O}_{7}$ & & 341 & $\begin{array}{l}323 ; 295 \\
255 ; 195 \\
\quad 159\end{array}$ & 305 & & {$[35,69]$} \\
\hline 45 & Nevadensin & $\mathrm{C}_{18} \mathrm{H}_{16} \mathrm{O}_{7}$ & 343 & & $328 ; 259$ & 313 & 269 & {$[45,69]$} \\
\hline 46 & Palmatine & $\mathrm{C}_{21} \mathrm{H}_{22} \mathrm{NO}_{4}$ & & 353 & $335 ; 235$ & $\begin{array}{l}317 \\
235 \\
137 \\
\end{array}$ & & {$[18,71]$} \\
\hline 47 & Hexose-hexose-N-acetyl & $\mathrm{C}_{14} \mathrm{H}_{25} \mathrm{NO}_{10}$ & 366 & & $186 ; 142$ & 142 & & {$[40]$} \\
\hline 48 & Fraxin & $\mathrm{C}_{16} \mathrm{H}_{18} \mathrm{O}_{10}$ & & 371 & $208 ; 352$ & 135 & & [29] \\
\hline 49 & $\begin{array}{l}\text { Fraxetin-7-O-beta- } \\
\text { glucuronide }\end{array}$ & $\mathrm{C}_{16} \mathrm{H}_{16} \mathrm{O}_{11}$ & & 385 & $\begin{array}{c}367 ; 272 ; \\
209 ; 175 \\
\quad 143\end{array}$ & 158 & & [73] \\
\hline 50 & Polydatin & $\mathrm{C}_{20} \mathrm{H}_{22} \mathrm{O}_{8}$ & 389 & & $227 ; 343$ & 184 & 143 & {$[46,60]$} \\
\hline
\end{tabular}




\begin{tabular}{|c|c|c|c|c|c|c|c|c|}
\hline 51 & Fucosterol & $\mathrm{C}_{29} \mathrm{H}_{48} \mathrm{O}$ & & 413 & $\begin{array}{c}395 ; 355 \\
271 ; 194 \\
\quad 119\end{array}$ & $\begin{array}{l}297 \\
199\end{array}$ & $\begin{array}{l}268 \\
187\end{array}$ & [30] \\
\hline 52 & Stigmasterol & $\mathrm{C}_{29} \mathrm{H}_{48} \mathrm{O}$ & & 413 & $\begin{array}{l}301 ; 259 \\
\quad 189\end{array}$ & 171 & & $\begin{array}{l}{[14,20,} \\
30]\end{array}$ \\
\hline 53 & Salvianolic acid D & $\mathrm{C}_{20} \mathrm{H}_{18} \mathrm{O}_{10}$ & 417 & & 373 & 329 & $\begin{array}{l}287 \\
209\end{array}$ & {$[21,22]$} \\
\hline 54 & Apigenin-7-O-glucoside & $\mathrm{C}_{21} \mathrm{H}_{20} \mathrm{O}_{10}$ & & 433 & $\begin{array}{c}414 ; 287 \\
186\end{array}$ & $\begin{array}{l}241 ; \\
158\end{array}$ & & $\begin{array}{c}{[15,17,} \\
29,34, \\
42,43, \\
58]\end{array}$ \\
\hline 55 & $\begin{array}{l}\text { Pelargonidin-3-O- } \\
\text { glucoside (callistephin) }\end{array}$ & $\mathrm{C}_{21} \mathrm{H}_{21} \mathrm{O}_{10}$ & & 433 & $\begin{array}{c}414 ; 271 \\
172\end{array}$ & $\begin{array}{l}172 \\
226\end{array}$ & 116 & $\begin{array}{l}{[23,59,} \\
65]\end{array}$ \\
\hline 56 & Phlorizin & $\mathrm{C}_{21} \mathrm{H}_{24} \mathrm{O}_{10}$ & & 437 & $397 ; 217$ & 377 & & $\begin{array}{l}{[26,29} \\
30,46 \\
53,57]\end{array}$ \\
\hline 57 & Catechin gallate & $\mathrm{C}_{22} \mathrm{H}_{18} \mathrm{O}_{10}$ & 441 & & $289 ; 169$ & $\begin{array}{l}245 \\
205\end{array}$ & 203 & {$[29,33]$} \\
\hline 58 & $\begin{array}{l}\text { Kaempferol-3-O- } \\
\text { galactoside }\end{array}$ & $\mathrm{C}_{21} \mathrm{H}_{20} \mathrm{O}_{11}$ & & 449 & 287 & $\begin{array}{l}269 \\
217\end{array}$ & & {$[25,29]$} \\
\hline 59 & $\begin{array}{l}\text { Eriodictyol-7- } O \text { - } \\
\text { glucoside }\end{array}$ & $\mathrm{C}_{21} \mathrm{H}_{22} \mathrm{O}_{11}$ & 449 & & 269 & $\begin{array}{l}207 \\
251\end{array}$ & 165 & $\begin{array}{l}{[16,29,} \\
42,63]\end{array}$ \\
\hline 60 & $\begin{array}{l}\text { Dihydrokaempferol } \\
\text { glucoside }\end{array}$ & $\mathrm{C}_{21} \mathrm{H}_{22} \mathrm{O}_{11}$ & 449 & & 287 & $\begin{array}{l}227 \\
269\end{array}$ & $\begin{array}{l}225 \\
149\end{array}$ & [46] \\
\hline 61 & Oleanoic acid & $\mathrm{C}_{30} \mathrm{H}_{48} \mathrm{O}_{3}$ & & 457 & $\begin{array}{c}439 ; 411 ; \\
365 ; 337 ; \\
293 ; 248 ; \\
205\end{array}$ & $\begin{array}{l}364 ; \\
337 ; \\
309 ; \\
219\end{array}$ & $\begin{array}{l}337 \\
319 \\
301 \\
279 \\
247 \\
232 \\
219\end{array}$ & {$[45,61]$} \\
\hline 62 & Ursolic acid & $\mathrm{C}_{30} \mathrm{H}_{48} \mathrm{O}_{3}$ & & 457 & $\begin{array}{l}411 ; 393 \\
365 ; 337 \\
279 ; 247\end{array}$ & $\begin{array}{l}365 ; \\
337 ; \\
292 ; \\
279 ; \\
247 ; \\
219 ; \\
205\end{array}$ & & $\begin{array}{l}{[20,44,} \\
61,69]\end{array}$ \\
\hline 63 & $\begin{array}{l}\text { Isorhamnetin 3-O- } \\
\text { rhamonoside }\end{array}$ & $\mathbf{C}_{22} \mathbf{H}_{22} \mathbf{O}_{11}$ & 461 & & $\begin{array}{l}315 ; 152 \\
219\end{array}$ & & & {$[52,53]$} \\
\hline 64 & Peonidin-3-O-glucoside & $\mathrm{C}_{22} \mathrm{H}_{23} \mathrm{O}_{11+}$ & & 463 & 301 & $\begin{array}{l}286 \\
258\end{array}$ & $\begin{array}{l}268 \\
258 \\
230 \\
202 \\
174 \\
121\end{array}$ & $\begin{array}{c}{[28,29} \\
37,59, \\
65]\end{array}$ \\
\hline 65 & Hyperoside & $\mathrm{C}_{21} \mathrm{H}_{20} \mathrm{O}_{12}$ & 463 & & $301 ; 179$ & $\begin{array}{l}257 \\
179\end{array}$ & $255 ; 147$ & $\begin{array}{c}{[13,25,} \\
26,29, \\
51,60, \\
63]\end{array}$ \\
\hline 66 & $\begin{array}{l}\text { Quercetin 3-O- } \\
\text { glucoside }\end{array}$ & $\mathrm{C}_{21} \mathrm{H}_{20} \mathrm{O}_{12}$ & & 465 & 303 & $\begin{array}{c}285 \\
257 \\
229 \\
201 \\
150\end{array}$ & $\begin{array}{l}229 \\
201 \\
155\end{array}$ & $\begin{array}{l}{[15,25,} \\
29,34, \\
43,46]\end{array}$ \\
\hline
\end{tabular}




\begin{tabular}{|c|c|c|c|c|c|c|c|c|}
\hline 67 & Taxifolin-3-O-glucoside & $\mathrm{C}_{21} \mathrm{H}_{22} \mathrm{O}_{12}$ & & 467 & $\begin{array}{c}449 ; 303 ; \\
188\end{array}$ & $287 ; 132$ & 260 & [29] \\
\hline 68 & $\begin{array}{l}\text { Quercetin-3-O- } \\
\text { glucuronide }\end{array}$ & $\mathrm{C}_{21} \mathrm{H}_{18} \mathrm{O}_{13}$ & 477 & & $301 ; 179$ & $273 ; 179$ & 151 & $\begin{array}{r}{[25,29,} \\
57,65]\end{array}$ \\
\hline 69 & $\begin{array}{l}\text { Isorhamnetin 3-O- } \\
\text { glucoside }\end{array}$ & $\mathrm{C}_{22} \mathrm{H}_{22} \mathrm{O}_{12}$ & & 479 & $317 ; 287$ & $301 ; 257$ & $\begin{array}{l}274 \\
228 \\
149\end{array}$ & $\begin{array}{l}{[15,25,} \\
29,52]\end{array}$ \\
\hline 70 & Myricetin-3-O-galactoside & $\mathrm{C}_{21} \mathrm{H}_{20} \mathrm{O}_{13}$ & 479 & & $299 ; 153$ & 271 & $\begin{array}{c}243 \\
171\end{array}$ & $\begin{array}{c}{[13,25,} \\
29,51, \\
63]\end{array}$ \\
\hline 71 & $\begin{array}{l}\text { Dimethylellagic acid } \\
\text { hexose }\end{array}$ & $\mathrm{C}_{22} \mathrm{H}_{20} \mathrm{O}_{13}$ & & 493 & 331 & $\begin{array}{c}299 \\
270 \\
242 \\
179 ; 150 \\
\end{array}$ & $\begin{array}{l}270 \\
225\end{array}$ & [59] \\
\hline 72 & Malvidin 3-O-glucoside & $\mathrm{C}_{23} \mathrm{H}_{25} \mathrm{O}_{12}$ & & 493 & 331 & $315 ; 179$ & $\begin{array}{l}315 \\
179\end{array}$ & $\begin{array}{l}{[29,37,} \\
65]\end{array}$ \\
\hline 73 & $\begin{array}{l}\text { 5-O-(4'-O-p-coumaroyl } \\
\text { glucosyl)quinic acid }\end{array}$ & $\mathrm{C}_{22} \mathrm{H}_{28} \mathrm{O}_{13}$ & & 501 & $\begin{array}{c}339 ; 277 \\
203\end{array}$ & 277 & & [34] \\
\hline 74 & $\begin{array}{l}\text { p- } \\
\text { Coumaroylcaffaoylquinic } \\
\text { acid }\end{array}$ & $\mathrm{C}_{25} \mathrm{H}_{24} \mathrm{O}_{11}$ & & 501 & $\begin{array}{l}355 ; 483 ; \\
181\end{array}$ & $\begin{array}{c}225 ; \\
281 ; \\
193 ; 120 \\
\end{array}$ & 133 & [61] \\
\hline 75 & Coumaric acid derivative & $\mathrm{C}_{30} \mathrm{H}_{30} \mathrm{O}_{7}$ & & 503 & $\begin{array}{l}457 ; 411 \\
382 ; 339 \\
\quad 293\end{array}$ & $\begin{array}{c}409 \\
391 ; \\
367 ; \\
339 ; 293\end{array}$ & $\begin{array}{l}323 \\
293 \\
233 \\
205\end{array}$ & [57] \\
\hline 76 & Malvidin acetyl hexoside & $\mathrm{C}_{25} \mathrm{H}_{28} \mathrm{O}_{13}$ & & 537 & $\begin{array}{l}331 ; 299 ; \\
261\end{array}$ & $\begin{array}{c}243 ; \\
211 ; \\
154 ; 111\end{array}$ & & [30] \\
\hline 77 & Procyanidin A-type dimer & $\mathrm{C}_{30} \mathrm{H}_{24} \mathrm{O}_{12}$ & & 577 & $\begin{array}{l}425 ; 397 \\
373 ; 287 \\
245 ; 181\end{array}$ & $\begin{array}{c}245 \\
218 \\
189 ; 123\end{array}$ & & $\begin{array}{r}{[13,29,} \\
51,61]\end{array}$ \\
\hline 78 & $\begin{array}{l}\text { Isovitexin 6"-O- } \\
\text { deoxyhexoside }\end{array}$ & $\mathrm{C}_{27} \mathrm{H}_{30} \mathrm{O}_{14}$ & & 579 & $\begin{array}{c}415 ; 297 \\
177\end{array}$ & $397 ; 344$ & 362 & [44] \\
\hline 79 & Vitexin 2"-O-glucoside & $\mathrm{C}_{27} \mathrm{H}_{30} \mathrm{O}_{15}$ & & 595 & $\begin{array}{l}415 ; 353 ; \\
283 ; 265\end{array}$ & 176 & & [44] \\
\hline 80 & $\begin{array}{l}\text { Kaempferol-3,7-Di-O- } \\
\text { glucoside }\end{array}$ & $\mathrm{C}_{27} \mathrm{H}_{30} \mathrm{O}_{16}$ & & 611 & $449 ; 287$ & $229 ; 165$ & $\begin{array}{c}213 \\
111\end{array}$ & {$[39,47]$} \\
\hline 81 & Cyanidin 3,5-O-dihexoside & $\mathrm{C}_{27} \mathrm{H}_{31} \mathrm{O}_{16}$ & & 611 & $287 ; 449$ & 287 & & {$[23,49]$} \\
\hline 82 & $\begin{array}{l}\text { Cyanidin 3,5-O- } \\
\text { diglucoside }\end{array}$ & $\mathrm{C}_{27} \mathrm{H}_{31} \mathrm{O}_{16}$ & & 611 & $287 ; 449$ & $\begin{array}{c}287 ; \\
269 ; \\
231 ; \\
199 ; 161\end{array}$ & $\begin{array}{l}231 \\
213 \\
189 \\
175 \\
147\end{array}$ & {$[37,65]$} \\
\hline 83 & $\begin{array}{l}\text { Apigenin 6-C-[6"-acetyl- } \\
\text { 2"-O-deoxyhexoside]- } \\
\text { glucoside }\end{array}$ & $\mathrm{C}_{29} \mathrm{H}_{32} \mathrm{O}_{15}$ & & 621 & $\begin{array}{l}561 ; 547 \\
\quad 461\end{array}$ & $\begin{array}{l}533 ; 461 \\
433\end{array}$ & 433 & [44] \\
\hline
\end{tabular}




\begin{tabular}{|c|c|c|c|c|c|c|c|}
\hline 84 & Peonidin-3,5-diglucoside & $\mathrm{C}_{28} \mathrm{H}_{33} \mathrm{O}_{16}$ & 625 & $301 ; 463$ & 286 & 258 & {$[37,65]$} \\
\hline 85 & $\begin{array}{l}\text { Malvidin 3-(6-O- } \\
\text { coumaroyl)glucoside }\end{array}$ & $\mathrm{C}_{32} \mathrm{H}_{31} \mathrm{O}_{14}$ & 639 & 331 & $\begin{array}{l}315 ; \\
299 ; \\
270 ; \\
242 ; \\
179 ; \\
150\end{array}$ & $\begin{array}{c}287 \\
270 \\
213 \\
179\end{array}$ & $\begin{array}{l}{[29,37,} \\
65]\end{array}$ \\
\hline 86 & $\begin{array}{l}\text { Petunidin 3-O- } \\
\text { glucoside-5-O-glucoside }\end{array}$ & $\mathrm{C}_{28} \mathrm{H}_{33} \mathrm{O}_{17}$ & 641 & $\begin{array}{l}317 ; 479 \\
420 ; 257\end{array}$ & 302 & $\begin{array}{l}274 \\
228\end{array}$ & {$[37,65]$} \\
\hline 87 & $\begin{array}{l}\text { Malvidin 3-(6'-p- } \\
\text { caffeoylglucoside) }\end{array}$ & $\mathbf{C}_{32} \mathbf{H}_{31} \mathbf{O}_{15}$ & 655 & $493 ; 331$ & $\begin{array}{l}315 \\
179\end{array}$ & $\begin{array}{l}313 \\
179\end{array}$ & {$[28,29]$} \\
\hline 88 & Malvidin 3,5-diglucoside & $\mathrm{C}_{32} \mathrm{H}_{31} \mathrm{O}_{15}$ & 655 & $331 ; 493$ & $\begin{array}{l}299 \\
179\end{array}$ & 281 & {$[37,65]$} \\
\hline 89 & $\begin{array}{l}\text { Cyclopassifloic acid } \\
\text { glucoside }\end{array}$ & $\mathrm{C}_{37} \mathrm{H}_{62} \mathrm{O}_{12}$ & 699 & $\begin{array}{c}537 ; 347 \\
271\end{array}$ & $\begin{array}{c}375 ; \\
259 ; \\
185\end{array}$ & & [44] \\
\hline
\end{tabular}

Research carried out using tandem mass spectrometry showed the presence of 89 biologically active compounds corresponding to the $V$. amurensis species. Salvianolic acids F, D and G, oleanolic, ursolic, myristoleic acids, berbericinin, mearnsetin, esculin, nevadensin, stigmasterol, fucosterol, Phlorizin, L-Tryptophan are identified for the first time in $V$. amurensis.

The identification of compounds ( $\mathrm{m} / \mathrm{z}$ values and fragmented ions) was carried out by comparing the obtained experimental data with known scientific results or mass spectrometric libraries. Anthocyanins have been identified in the extracts: Malvidin-3-Oglucoside, Pelargonidin-3-O-glucoside (callistephin), Peonidin-3-O-glucoside, Cyanidin3,5-dihexoside, Cyanidin-3,5-diglucoside, Peonidin-3,5-diglucoside, Malvidin 3-(6-Ocoumaroyl) glucoside, Petunidin-3-O-glucoside-5- $O$-glucoside, Malvidin 3-(6'- $p$-caffeoyl glucoside), Malvidin 3,5-diglucoside. Obtained mass spectrometry data correlate with scientific sources [13, 23, 28, 29, 37, 49, 51, 59, 61, 65]. A large group of flavonoids identified; flavonols Kaempferol, Aromadendrin, Kaempferide, Quercetin, Dihydroquercetin, Kaempferol-3-O-galactoside, Quercetin 3- $O$ - galactoside, Taxifolin-3-Oglucoside, Quercetin-3-O-glucuronide, Isorhamnetin-3- $O$-rhamonoside, Isorhamnetin-3-Oglucoside, Myricetin-3- $O$-galactoside, Kaempferol-3,7-Di- $O$-glucoside [13, 24, 25, 29, 41, 42, 45, 51, 54, 56, 57, 60, 62, 64, 65, 68, 70, 74]; flavones: Apigenin, Luteolin, Nevadensin, Apigenin-7-O-glucoside, Isovitexin 6"-O-deoxyhexoside, Vitexin 2"-O-glucoside, Apigenin 6-C-[6"-acetyl-2"- $O$-deoxyhexoside]-glucoside $[13,15,17,34,43,44,45,58,60,67,69]$; flavanones: Naringenin, Hesperitin, Eriodictyol-7-O-glucoside [16, 29, 42, 53, 60, 62, 63]; Flavan-3-ols: Catechin, Epicatechin [13, 25, 29, 46, 53, 57, 60].

Glycosylated coumarins have also been identified: Umbelliferone, Esculin, Fraxin, Fraxetin-7-O-beta-glucuronide [29, 30, 34, 36, 71,73], berberine Palmatine [18, 71], stilbenes Polydatin and trans-Resveratrol, [30, 46, 60], sterols: Fucosterol, Stigmasterol [14, 20, 30], dihydrochalcone Phlorizin [26, 29, 30, 46, 53, 57].

It should be noted that compounds such as coumarins Umbelliferone, Fraxin and Esculin, flavone Nevadensin, flavan-3-ol Epicatechin, sterol Fucosterol, flavanol Taxifolin-3-Oglucoside were identified by mass spectrometry only in island samples of wild grapes. $V$. amurensis (Pakhtusov Islands and Rikord Island, Peter the Great Bay, Sea of Japan). 


\section{Conclusions}

Amur grape $V$. amurensis Ruprecht contains a large number of polyphenolic complexes, which are biologically active compounds. In this work, we have tried for the first time to conduct a comparative metabolic study of biologically active substances of wild grapes obtained from five different places in the Primorsky and Khabarovsk territories. HPLC in combination with a BRUKER DALTONIKS ion trap (tandem mass spectrometry) was used to identify target analytes in extracts. The results showed the presence of 89 biologically active compounds corresponding to the species $V$. amurensis, and Salvianolic acids F, D and G, Oleanolic, Ursolic, Myristoleic acids, Berbericinin, Mearnsetin, Esculin, Nevadensin, Stigmasterol, Fucosterol, Phlorizin, L-Tryptophan were identified for the first time in $V$. amurensis.

The findings may support future research into the production of various pharmaceutical and dietary supplements containing $V$. amurensis extracts. A wide variety of biologically active polyphenolic compounds opens up rich opportunities for the creation of new drugs and biologically active additives based on extracts from this family of grapes (Vitaceae).

\section{References}

1. Ampelography USSR, 494 (1946)

2. N.I. Vavilov, Origin and geography of cultivated plants, 440 (1987)

3. E.W. Berry, Lower Cretaceous. Baltimore, Maryland Geological Survey (1911)

4. E.W. Berry, Maryland Geological Survey (1917)

5. A. N. Krishtofovich, Paleontological history of grapes, 23(5-6), 365 (1938)

6. Krishtofovich A. N. Sarmatian flora of the Krynka river, 98, 5 (1931)

7. A. N. Krishtofovich, M. I. Borsuk, Miocene plants from the Irtysh River near the city Tara in Western Siberia, 5, 375 (1939)

8. G. Hegi Vitaceae, Rebengewachse. Illustrierte Flora von Mittel-Europa. Munchen, 5(1), 350 (1925)

9. I.V. Michurin, The results of half a century of work on the development of new varieties of fruit plants, I-II (1929)

10. I.V. Michurin, Bulletin of gardening, fruit growing and horticulture, 4-5 (1907)

11. V.G. Sprygin, N.F. Kushnerova, S.E. Fomenko, T.N. Gordeychuk, E.E. Solodova, Respiratory Physiology and Pathology Bulletin, 11, 1 (2002)

12. State Pharmacopeia XIV (2018) https://pharmacopoeia.ru

13. G. Abeywickrama, S.C. Debnath, P. Ambigaipalan, F. Shahidi, Phenolics of selected cranberry genotypes and their antioxidant efficacy (2016)

14. D. Bakir, M. Akdeniz, A. Ertas, M.A. Yilmaz, I. Yener, M. Firat, U. Kolak, Food Biochem (2020)

15. L. Barros, M. Duenas, A.M. Carvalho, Food and Chem. Toxicol, 50(5), 1576 (2012)

16. A. Bodalska, A. Kowalczyk, M. Wlodarczyk, I. Feska, Analysis of Polyphenolic Composition of a Herbal Medicinal Product-Peppermint, 25, 69 (2020)

17. C. Carazzone, D. Mascherpa, G. Gazzani, A. Papetti, Food Chem, 138, 1062 (2013)

18. D.S.A. Cassiano, I.M.A. Reis, I. de Oliveira Estrela, H.F. de Freitas, S.S. da Rocha Pita, J.M. David, A. Branco, Comp. Biol. Chem, 83, 107129 (2019) 
19. W. Chen, L. Gong, Z. Guo, W. Wang, H. Zhang, X. Liu, S. Yu, L. Xiong, J. Luo, Molecular Plant, 6(6), 1769 (2013)

20. X. Chen, P. Zhu, B. Liu, D. Ge, L. Wei, Y. Xu, J. Pharm. Biomed. Anal, 159, 490 (2018)

21. X. Chen, S. Zhang, Z. Xuan, D. Ge, X. Chen, J. Zhang, Q. Wang, Y. Wu, B. Liu, The Cells. Molecules, 22, 811 (2017)

22. M. Cirlini, P. Mena, M. Tassotti, K.A. Herrlinger, K. Nieman, C. Dall'Asta, D. Del Rio, Molecules, 21, 1007 (2016)

23. L.P. da Silva, E. Pereira, T.C.S.P. Pires, M.J. Alves, O.R. Pereira, L. Barros, I.C.F.R. Ferreira, Food Res. Int, 119, 34 (2019)

24. Daikonya, A.; Kitanaka, S. Constituents isolated from the roots of Rhodiola sacra S. H. Fu. Japan. J Food Chem Safety, 18(3), 183 (2011)

25. M. De Rosso, L. Tonidandel, R. Larcher, G. Nicolini, A. Dalla Vedova, F. De Marchi, M. Gardiman, M. Giust, R. Flamini, Food Chem, 163, 244 (2014)

26. Z. Fan, Y. Wang, M. Yang, J. Cao, A. Khan, G. Cheng, Food Chem, 318, 126512 (2020)

27. T. Farrell, L. Poquet, F. Dionisi, D. Barron, G. Williamson, J. Pharm. Biomed. Anal, 55, 1245 (2011)

28. M. Garg, M. Chawla, V. Chunduri, R. Kumar, S. Sharma, A. Sharma, N.K. Kaur, N. Kumar, J.K. Mundey, M.K. Saini, S.P. Singh, J. Cereal Sci, 71, 138e144 (2016)

29. P. Goufo, R.K. Singh, I. Cortez, Antioxidants, 9, 398 (2020)

30. A.R. Hamed, S.S. El-Hawary, R.M. Ibrahim, U.R. Abdelmohsen, A.M. El-Halawany, Bioassay Guided Approach. J. Chrom. Sci, 1 (2020)

31. F. Han, Y. Li, L. Ma, T. Liu, Y. Wu, R. Hu, A. Song, R. Yin, Talanta, 160, 183 (2016)

32. V. Ivanova-Petropulos, Z. Naceva, V. Sandor, L. Makszin, L. Deutsch-Nagy, B. Berkics, T. Stafilov, F. Kilar, Electrophoresis, 39, 1597 (2018)

33. R. Jaiswal, L. Jayasinghe, N. Kuhnert, J. Mass Spectrom, 47, 502 (2012)

34. R. Jaiswal, H. Muller, A. Muller, M.G.E. Karar, N. Kuhnert, Phytochem, 108, 252 (2014)

35. R.-W. Jiang, K.-M. Lau, P.-M. Hon, T.C.W. Mak, K.-S. Woo, K.-P. Fung, Current Med. Chem, 12, 237 (2005)

36. S. Kim, S. Oh, H.B. Noh, S. Ji, S.H. Lee, J.M. Koo, C.W. Choi, H.P. Jhun, L. Roots. Molecules, 23, 3001 (2018)

37. E.S. Lago-Vanzela, R. Da-Silva, E. Gomes, E. Garcia-Romero, E. Hermosin-Gutierres, Agric. Food Chem., 59, 13136 (2011)

38. R. Lang, N. Dieminger, A. Beusch, Y.-M. Lee, A. Dunkel, B. Suess, T. Skurk, A. Wahl, H. Hauner, T. Hofmann, Anal. Bioanal. Chem. Sci., 8(08), 116 (2018)

39. G. Le Gall, M.S. DuPont, F.A. Mellon, A.L. Davis, G.J. Collins, M.E. Verhoeven, I.J. Colquhoun, J. Agricult. Food Chem, 51, 2438 (2003)

40. T. Levandi, T. Pussa, M. Vaher, A Ingver, R. Koppel, M. Kaljurand, Food Chem, 63(1), 86 (2014)

41. T.H. Lee, C.C. Hsu, G. Hsiao. J.Y. Fang, W.M. Liu, C.K. Lee, Planta Med., 82(8), 698 (2016)

42. X. Li, T. Tian, Frontiers in Pharm, 9, 1067 (2018)

43. M.M. Marzouk, S.R. Hussein, A. Elkhateeb, M. El-shabrawy, E.-S. S. Abdel-Hameed, S.A. Kawashty, J. Applied Pharm. Sci., 8(08), 116 (2018) 
44. M. Ozarowski, A. Piasecka, A. Paszel-Jaworska, A. D. Siqueira de Chaves, A. Romaniuk, M. Rybczynska, A. Gryszczynska, A. Sawikowska, P. Kachlicki, P.L. Mikolajczak, A. Seremak-Mrozikiewicz, Braz. J. Pharmacol, 28, 179 (2018)

45. R. Pandey, B. Kumar, J. Liquid Chromatogr. \& Related Technol, 39, 225 (2016)

46. L. Paudel, F.J. Wyzgovski, J.C. Scheerens, A.M. Chanon, R.N. Reese, D. Smiljanic, C.; C. Wesdemiotis, J.J. Blakeslee, K.M. Riedl, P.L. Rinaldi, J. Agricult. Food. Chem, 61, 12032 (2013)

47. A. Petsalo, J. Jalonen, A. Tolonen, Chromatogr, 1112(1-2), 224 (2006)

48. S. Piccolella, G. Crescente, M.G. Volpe, M. Paolucci, S. Pacifico, Molecules. Chem, 24, 3630 (2019)

49. P.C. Pradhan, S. Saha, J Food Sci. Technol, 53(2), 1205 (2016)

50. P. Quifer-Rada, A. Vallverdu-Queralt, M. Martinez-Huelamo, G. Chiva-Blanch, O. Jauregui, R. Estruch, R. Lamuela-Raventos, Food Chem, 169, 336 (2015)

51. N. Rafsanjany, J. Senker, S. Brandt, U. Dobrindt, A. Hensel, J. Agric. Food Chem, 63, 8804 (2015)

52. S.A.O. Santos, C.S.R. Freire, M.R.M. Domingues, A.J.D. Silvestre, C.P. Neto, J. Agric. Food Chem, 59, 9386 (2011)

53. S.A.O. Santos, C. Vilela, C.S.R. Freire, C.P. Neto, A.J.D. Silvestre, J. Chromatogr. B. 938, 65 (2013)

54. A.J. Seukep, Y.-L. Zhang, Y.-B. Hu, M.-Q. Guo, Pharmaceut, 13, 59 (2020)

55. K. Schoedl, A. Forneck, M. Sulyok, R. Schuhmacher, J. Agric. Food. Chem, 59, 10787 (2011)

56. M. Sharma, R. Sandhir, A. Singh, P. Kumar, A. Mishra, S. Jachak, S.P. Singh, J. Singh, J. Roy, Front. Plant. Sci., 7, 1870 (2016)

57. V. Spinola, J. Pinto, P.C. Castilho, Food Chemistry, 173, 14 (2015)

58. M. Suarez, A. Macia, M.-P. Romero, M.-J. Motiva, J. Chromatogr. A., 1214, 90 (2008)

59. J. Sun, X. Liu, T. Yang, J. Slovin, P. Chen, Food Chem, 146, 289 (2014)

60. J. Sun, F. Liang, Y. Bin, P. Li, C. Duan, Molecules, 12, 679 (2007)

61. L. Sun, S. Tao, S. Molecules, 24, 159 (2019)

62. A. Vallverdu-Queralt, O. Jauregui, A. Medina-Remon, R.M. Lamuela-Raventos, Food Chem., 60, 3373 (2012)

63. M.N. Viera, P. Winterhalter, G. Jerz, Phytochem. Anal., 27, 116 (2016)

64. I.V. Voskoboinikova, N.A. Tjukavkina, S.V. Geodakyan, Y.A. Kolesnik, V.K. Kolhir, V.A. Zjuzin, S.J. Sokolov, Phytotherapy Res., 7, 208 (1993)

65. H. Wang, E.J. Race, A.J. Shrikhande, Agric. Food Chem., 51, 1839 (2003)

66. S. Wang, C.Q. Fan, P. Wang, J. Chromatogr. B., 981, 1 (2015)

67. A. Wojakowska, J. Perkowski, T. Goral, M. Stobiecki, J. Mass. Spectrom, 48, 329 (2013)

68. J. Xiao, T. Wang, P. Li, R. Q. Liu, Li, K. Bi, J. Chromatogr. B., 1028, 33 (2016)

69. L. L. Xu, J. J. Xu, K. R. Zhong, Z. P. Shang, F. Wang, R. F. Wang, B. Liu, Molecules, 22, 1756 (2017)

70. X. Xu, B. Yang, D.Wang, Y. Zhu, X.Miao, W. Yang, Molecules, 25, 4612 (2020)

71. L. Yang, X. Meng, X. Yu, H. Kuang, J. Pharm. Biomed. Analys, 134, 43 (2017)

72. S.T. Yang, X. Wu, W. Rui, J. Guo, Y.F. Feng, Acta Chromatographica, 27, 711 (2015) 
73. T. Yasuda, M. Fukui, T. Nakazawa, A. Hoshikawa, K. Ohsawa, J. Nat. Prod., 69, 755 (2006)

74. W.-H. Zhang, I.-Ch. Chao, D.-J. Hu, F. Shakerian, J. AOAC Int., 102(3) (2019) 